\title{
WIZARDS OF MOTION: PROMOTING SCIENCE, ENGINEERING AND TECHNOLOGY IN THE SCHOOLS
}

McPherson, M N; Zerpa, C E; Montelpare, W J

School of Kinesiology, Lakehead University, Ontario Canada

$$
\text { moira.mcpherson@lakeheadu.ca }
$$

\section{INTRODUCTION}

The Natural Sciences and Engineering Research Council's (NSERC) PromoScience program provides support for organizations working with young Canadians to promote an understanding of science, engineering, and technology. Lakehead University's Wizards of Motion program was developed to provide an interactive introduction to the science of human motion. The purpose of this presentation is to highlight two modules specifically designed to link biomechanics with the school science curriculum.

Biomechanics is an area of study that examines the forces acting on the human body and the effects produced by those forces. Quantitative approaches examine the motion characteristics of human movement using both kinematic and kinetic parameters. Data collection is followed by rigorous data processing and analysis techniques. As part of the Wizards of Motion program, scientific and technical experts visited Ontario classrooms with dynamic multimedia presentations and portable laboratory experiences for elementary school students. The Wizards of Motion curriculum links closely to the Ministry of Ontario science curriculum but is expanded to include human motion applications and hands-on quantitative data experiences.

\section{THE HEAD SAFETY MODULE}

The Head Safety Module was designed to be delivered to grade 7 students during a single two hour block in regular school hours. The program includes an introduction using a multimedia presentation combined with technology demonstrations to help capture the student's attention while presenting concepts and terms such as biomechanics, force, impulse, shock absorption and safe helmet design and practice. Students use a custom designed Head Impact Measurement System (Figure 1) to simulate falls and subsequent head impact. The measurement system is comprised of a support frame and a mounted head-form with attached linear accelerometers. The device is interfaced to a laptop computer for analogue to digital data conversion. A lab activity enables students to observe and compare impact measurements associated with head trauma. At the end of the program the students were expected to:

- Define and identify basic biomechanical terms and concepts, determinants of head injury and safe practices.

- Discuss the characteristics of materials used to dampen or absorb force.

- Generate and interpret data from the Head Impact Measurement System and relate the finding to the design of protective helmets.

- Discuss the specifications of helmets used to protect the head while cycling.

- Articulate an increased interest in practicing safe helmet-use behaviors.

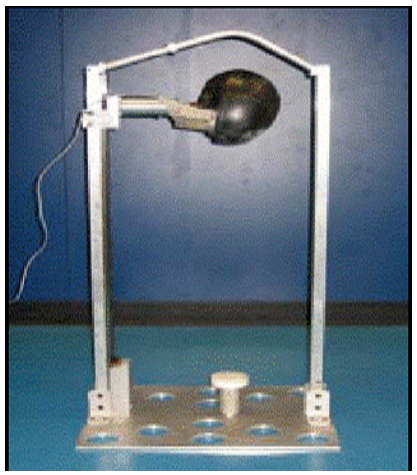

Figure 1: The Head Impact Measurement System

\section{EVALUATION OF THE HEAD SAFETY MODULE}

The module has been delivered in over 30 classrooms and in each case a standard evaluation form was used to capture student and teacher comments. The results continue to indicate that the module engages students in exploring science and technology through fun, dynamic presentations and activities. A study ${ }^{1}$ conducted to evaluate students' head safety knowledge and attitudes toward helmet use following participation in the program, showed a significant pre-post change in level of knowledge scores for the intervention group. Students indicated an increase in their intention to wear a helmet in the future. Comments provided by the students and the teachers suggested that the program enabled observation and comparison of kinematic and kinetic variables associated with head trauma based on impact.

\section{THE ELECTROMYOGRAPHY (EMG) MODULE}

A newly designed EMG module introduces the concepts of neural stimulation leading to muscle contraction and is based on the relationship between the electrical activity across the muscle membrane and force. Following the introduction to force measurement, muscle physiology, and signal processing, students construct an electronic circuit (Figure 2). The circuit is designed to examine force production while contracting the flexor digitorum superficialis muscle. The system uses an electronic circuit to convert the mechanical force applied by the fingers pushing on a switch to an electrical signal shown by the intensity of a light emitting diode. A measure of the amount of force is displayed using a voltmeter. Working in small groups, students record values for a series of trials and plot and discuss the data.

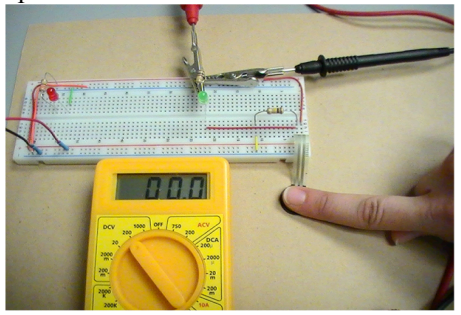

Figure 2: Electronic circuit to measure force

A portable EMG system is then used to measure the electrical activity associated with the mechanical action of squeezing on a paper clip. Students acquire the raw signal which is then processed using both full wave rectification and integration (Figure 3). The EMG output is displayed and printed for each group of students. Examples of control of voluntary and reflexive movement applications are drawn. A review of the curriculum is planned following the first year of delivery.

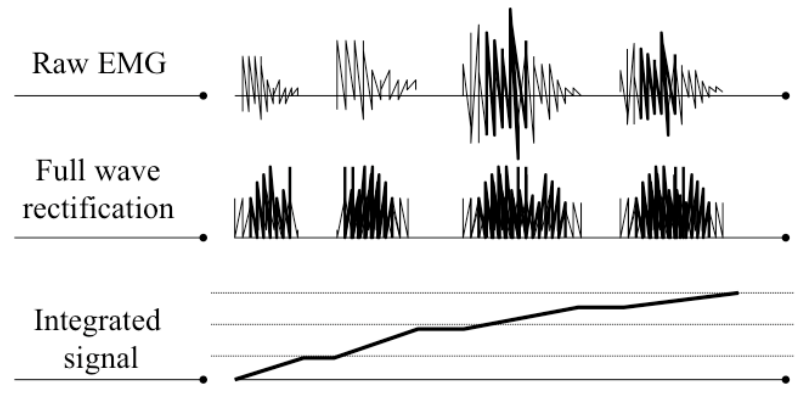

Figure 3: Raw and processed EMG signal

\section{SUMMARY}

Throughout the delivery of each of the Wizard modules a significant effort has been made to motivate youth to consider pursuing post secondary education in science and technology while promoting health messages in school. Further evaluation of the impact of the program is recommended.

\section{REFERENCES}

1. McPherson MN, Marsh PK, Montelpare, WJ, Van Barneveld C, Zerpa, C. Am J Health Educ. 2009; 40(2) 71-79. 